\title{
Sirt6 mRNA-incorporated endothelial microparticles (EMPs) attenuates DM patient-derived EMP-induced endothelial dysfunction
}

\author{
Tong Jing ${ }^{1, *}$, Kuang Ya-Shu ${ }^{1, *}$, Wang Xue-Jun ${ }^{1}$, Hou Han-Jing ${ }^{1}$, Lai Yan ${ }^{1}$, Yao Yi- \\ $\mathrm{An}^{1}{ }^{1}$ Chen Fei ${ }^{1, * *}$ and Liu Xue-Bo ${ }^{1, * *}$ \\ ${ }^{1}$ Department of Cardiology, Shanghai Tongji Hospital, Tongji University, Shanghai, China \\ *These authors have contributed equally to this work \\ ${ }^{* *}$ Co-corresponding author \\ Correspondence to: Chen Fei, email: riverapt@126.com \\ Liu Xue-Bo, email: liuxb70@hotmail.com \\ Keywords: EMPs; sirtuin 6; endothelial dysfunction; diabetes; vascular complication \\ Received: June 15,2017 Accepted: December 03, $2017 \quad$ Published: December 15, 2017 \\ Copyright: Jing et al. This is an open-access article distributed under the terms of the Creative Commons Attribution License 3.0 \\ (CC BY 3.0), which permits unrestricted use, distribution, and reproduction in any medium, provided the original author and source \\ are credited.
}

\section{ABSTRACT}

Background: Endothelial microparticles (EMPs) are small vesicles released by endothelial cells (ECs); they are considered biomarkers for endothelial dysfunction and therapeutic targets in diabetes-related vascular disease. Sirtuins have also been shown to play important roles in diabetes by regulating endothelial dysfunction. However, the effect of sirtuin-incorporated EMPs on their parental ECs remains unknown.

Aim: The present study aims to investigate the diagnostic value of EMPs in diabetes and detect the protective effects of sirtuin 6 (Sirt6) mRNA -incorporated EMPs on endothelial dysfunction.

Methods: EMPs were prepared from cultured HUVECs and venous blood from patients with diabetes $(n=10)$ and from healthy volunteers $(n=6)$ after sequential centrifugation. Adv-Sirt6 or Sirt6 siRNA was used to alter Sirt6 expression. EC angiogenesis, inflammatory phenotypes, nitric oxide (NO) formation and eNOS phosphorylation were used to evaluate endothelial dysfunction.

Results: The levels of EMPs in diabetic patients and high glucose-cultured HUVECs are high, whereas Sirt6 expression in plasma and EMPs is low. EMPs generated from diabetic patients or high glucose-cultured HUVECs increase inflammatory chemokine release and blunt EC angiogenesis. Furthermore, EMPs enriched with Sirt6 mRNA induces EC angiogenesis, increases eNOS phosphorylation and impedes inflammatory chemokine release. Inhibition of Sirt6 mRNA expression in EMPs by siRNA hinders angiogenesis and eNOS phosphorylation but increases cellular inflammation.

Conclusion: The Sirt6 mRNA-carrying EMPs may ameliorate endothelial dysfunction in diabetic patients.

\section{INTRODUCTION}

The incidence of diabetes mellitus (DM) is prevalent in both developed and developing countries. As of 2035 , an estimated 592 million individuals worldwide will be diagnosed with DM, which is increased from 387 million in 2014 [1]. DM-induced macrovascular and microvascular complications lead to reduced quality of life, added health care costs and even early death [2]. It is noteworthy that vascular endothelial dysfunction is the main factor in the pathogenesis of diabetic vascular complications via increased inflammation, dysregulated 
blood flow, cellular trafficking, decreased nitric oxide (NO) production and the induction of endothelial cell death, thus contributing to increased mortality rates $[3,4]$. Consequently, the comprehensive analysis and amelioration of endothelial impairment may inhibit the progression of DM-related vascular complications.

Endothelial dysfunction is associated with inordinate intercellular information exchange between endothelial cells (ECs) and immune cells, smooth muscle cells, fibroblasts or even parent ECs [5]. In addition, intercellular information exchange may involve the release of extracellular vesicles, which can function as intercellular carriers of ligands, enzymes, RNA and miRNA [6]. However, the precise mechanism of intercellular information exchange between ECs in DM remains largely unknown.

Recently, documents have verified that endothelial microparticles (EMPs) are novel, complex membrane-shed vesicles that facilitate endothelial dysfunction in DMrelated vascular complications [2]. EMPs range from 0.1-1 $\mu \mathrm{m}$ in size and are generated from ECs during apoptosis, activation or injury [7]. Importantly, previous data reveal that the biological content (protein, lipid, miRNA and mRNA) and functional effect of EMPs depend on the condition of the ECs from which they are released [8]. Hyperglycemic conditions significantly increase EMP expression, which is low under normal conditions, and subsequently change their biological effect on target cells [9]. Under DM conditions, microparticles (MPs) have shown noxious effects on ECs in a previous study $[6,7$, 10]. in vivo studies have shown that endothelial cells or platelet derived-MPs impair angiogenesis, reduce cerebral microvascular density and accelerate the progression and severity of retinopathy in DM; meanwhile, MPs, especially CD31+ MPs, mediated EC dysfunction in ex vivo studies [10-12]. In addition, EMPs also act as vectors during intercellular information exchange though coupling with membrane-associated receptors, releasing signaling molecules, such as active proteins and miRNA, and finally, mediating adaptive responses [13], but the effects of EMPs on their parent ECs are still unclear. Therefore, the present study aims to understand the release levels of EMPs and they cause endothelial dysfunction in DM and high glucose mediated-HUVECs.

Sirtuins (Sirts), a family of evolutionarily conserved nicotinamide adenine dinucleotide-dependent histone deacetylases, contains 7 Sirt genes, which have been tested to participate in multiple pathophysiological processes, such as DNA damage repair, apoptosis, senescence and neurodegeneration [14]. Recently, mounting evidence suggests that Sirts exerts effects in multiple cardiovascular disease models, including atherosclerosis, cardiac hypertrophy, chronic inflammation and vascular aging [15]. Additionally, previous studies have revealed that Sirt6 expression is downregulated in response to DM. Moreover, Sirt6 deficiency exacerbates the DM-induced impairment of wound healing and endothelial senescence [16, 17]. However, Sirt6 expression in EMPs remains unknown. This study aims to investigate the possible roles of Sirt6containing EMPs in endothelial dysfunction in DM.

\section{RESULTS}

\section{Increased plasma EMPs levels and reduced Sirt6 expression in DM patients}

When compared with the healthy group, plasma CD31+/CD42- EMPs levels were significantly increased in the DM group (Figure 1A). Meanwhile, CD62+/CD42EMP levels were augmented in DM patients (Figure 1B). However, the levels of CD42+ MPs were not significantly different between the two groups (Figure 1C). This indicated the increased release of EMPs into plasma in DM patients.

Decreased expression of Sirt6 is a widely accepted risk factor of DM and atherosclerosis. Therefore, we speculated that EMP-mediated injury may be caused by Sirt6 deficiency. In this study, we first detected the expression of plasma Sirt6 using ELISA. The results revealed that Sirt6 expression in plasma from DM patients was significantly reduced compared with plasma from healthy volunteers (Figure 1D and Supplementary Figure 1A). Then, we measured Sirt6 mRNA or protein expression in EMPs. In contrast to Sirt6 $m R N A$, immunoblot experiments revealed nearly no expression of Sirt6 protein in different EMPs (Figure 1E), suggesting that Sirt6 $m R N A$ is selectively packaged in EMPs. Additionally, we noted that Sirt6 $m R N A$-containing EMPs were obviously decreased in DM patients (Figure 1F). These data indicated that Sirt6-containing EMPs may play important roles in DM patients.

To estimate the EMPs and plasma Sirt6 levels whether related with blood glucose levels, we examined the blood glucose levels in DM patients and found that there was significant difference in 2-hour post-meal blood glucose but not in fasting blood-glucose. Additionally, the increased EMP levels and decreased Sirt6 levels were observed in patients with high blood-glucose levels accordingly (Figure $1 \mathrm{G})$.

\section{EMPs from DM patients induced EC dysfunction ex vivo}

As previous reported, the level of EMP at $1 \times 10^{5}$ EMPs/ml is sufficient to generate effects for target cells [9], therefore we applied EMPs at $1 \times 10^{5} \mathrm{EMPs} / \mathrm{ml}$ to cultured HUVECs. As shown in Figure 2A, the plasma EMPs could effectively be engulfed by cultured HUVECs. Then, we measured $I L-1 b, T N F-a$ and $I L-10$ mRNA levels after EMP stimulation. We found that DM patientderived EMPs significantly induced $I L-1 b$ and $T N F-a$ mRNA expression, whereas they inhibited $I L-10$ mRNA 
expression compared to healthy volunteer-derived EMPs (Figure 2B), which indicated that DM patient-derived EMPs increased inflammatory release in cultured ECs. Additionally, we also measured NO expression. As shown in Figure 2C, DM patient-derived EMPs decreased NO expression in ECs. Combining these data, we deduced that DM patient-derived EMPs induced EC dysfunction ex vivo.

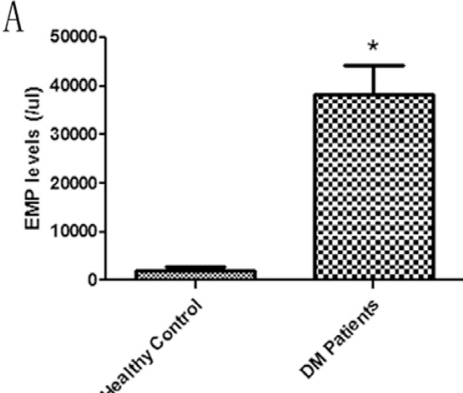

CD31+/CD42-EMPs

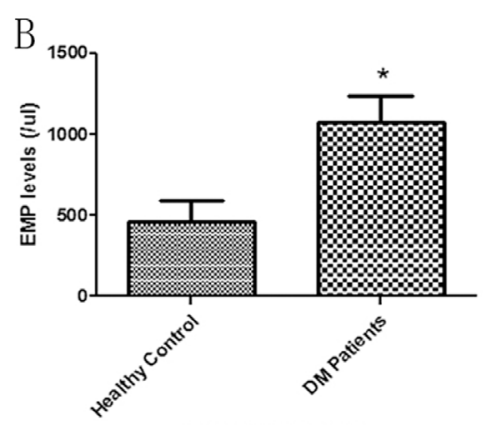

CD62+/CD42- EMPs

D

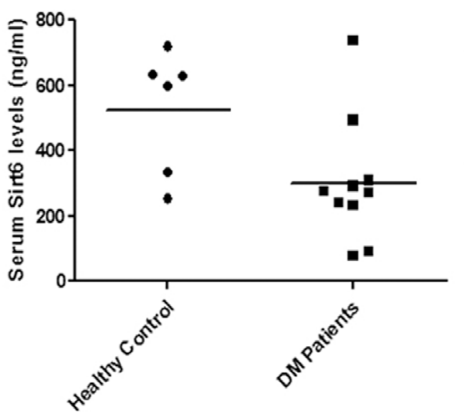

E

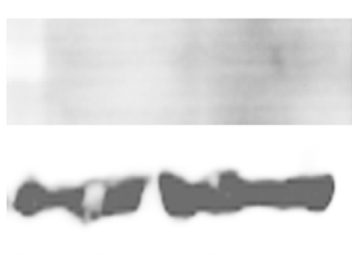

HCT EMPS DM EMPS $\operatorname{sirt6}$

$\beta-\operatorname{actin}$

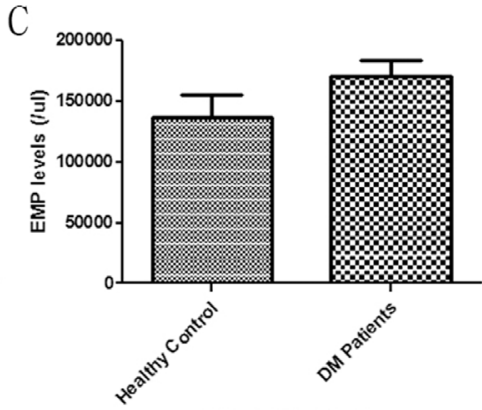

CD31-/CD42- EMPs

F

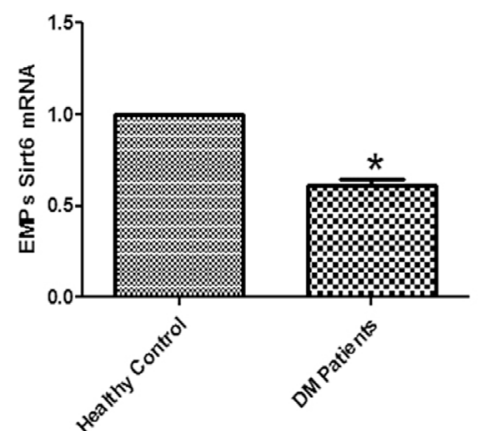

G
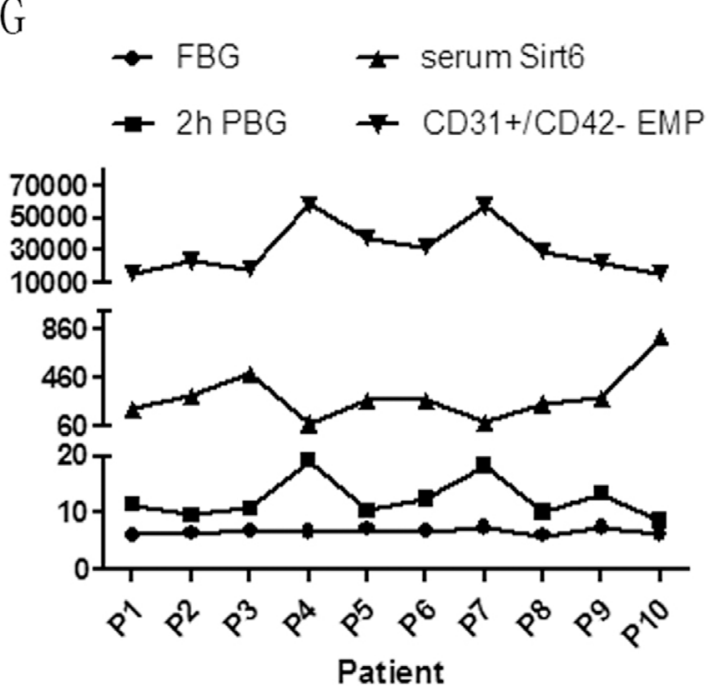

Figure 1: EMP levels and Sirt6 expression in DM patients. (A) Increased apoptosis-derived EMP (CD31+) levels in DM patients compared to healthy controls. (B) Increased activated EMP (CD62+) levels in DM patients compared to healthy controls. (C) There is no difference in platelet-derived (CD42+) MPs between healthy controls and DM patients. (D) Decreased serum Sirt6 levels in DM patients. (E) There is no Sirt6 protein in EMPs. (F) Decreased incorporation of Sirt6 $m R N A$ in DM patient-derived EMPs compared to EMPs from healthy controls. (G) Boosted CD31+ EMP levels related with decreased serum Sirt6 levels and increased 2-hour post-meal blood glucose levels. Healthy control, $\mathrm{n}=6$; and DM, $\mathrm{n}=10$. EMPs, endothelial microparticles; MPs, microparticles; HCT, healthy control; DM, diabetes mellitus; FBG, fast blood glucose; $2 \mathrm{~h}$ PBG, 2-hour post-meal blood glucose; $\mathrm{p}(\mathrm{n})$, patient numble. ${ }^{*} \mathrm{p}<0.05$. GAPDH mRNA serves as an internal control. 


\section{High glucose levels promoted EMP release $e x$ vivo}

As we shown in previous document [18], 33 $\mathrm{mM}$ glucose had sufficient effect to induce HUVECs dysfunction, therefore, we directly applied $33 \mathrm{mM}$ glucose to cultured HUVECs to verify the relationship between DM and EMP release. As expected, high glucose levels increased CD31+ EMP production (Figure 3A). Additionally, we detected the expression of Sirt6 $m R N A$ and protein in EMPs and HUVECs. As with DM patients, high glucose levels inhibited Sirt6 mRNA and protein expression in EMPs and ECs (Figure 3B, 3C and Supplementary Figure 1B).

\section{Sirt6 $m \boldsymbol{R} N \boldsymbol{A}$-containing EMPs control EMP- mediated HUVEC dysfunction ex vivo}

As Sirt6 $m R N A$ expression decreased in DM patientor high glucose-derived EMPs, we suspected that Sirt6 deficiency may be related to EMP-mediated HUVEC dysfunction. First, we applied Sirt6 siRNA or Adv-Sirt6 to generate Sirt6-deficient or Sirt6-enriched EMPs (Figure 4A, 4B). Then, we added these EMPs to culturedHUVECs. Interestingly, Sirt6-deficient EMPs effectively mimicked high glucose- derived EMPs and mediated EC dysfunction, including increased inflammatory cytokine production and decreased NO production, whereas Sirt6enriched EMPs reversed the high glucose-derived EMPmediated EC dysfunction (Figure 4C-4E). Angiogenesis is a very important sign of ECs favorable function, therefore we added EMPs to HUVECs. As shown in Figure 4I and Supplementary Figure 1D, high glucose and Sirt6$m R N A$-deficient EMPs impeded HUVECs tube formation, whereas Adv-Sirt6-treated EMPs induced tube formation in high glucose-cultured HUVECs.

To prove that mRNAs incorporated in the EMPs are mature and complete Sirt6 $m R N A$, we then measured the EMPs Sirt6 $m R N A$ sequence using RNAseq. As shown in Figure 4F and Supplementary Figure 2, the Sirt6 mRNAs incorporated in the EMPs are mature. To further confer the Sirt6 $m R N A$ incorporated in the EMPs are functional, we added Sirt6 $m R N A$-enriched EMPs to Sirt6 knock down (KD) HUVECs, which induced by
A
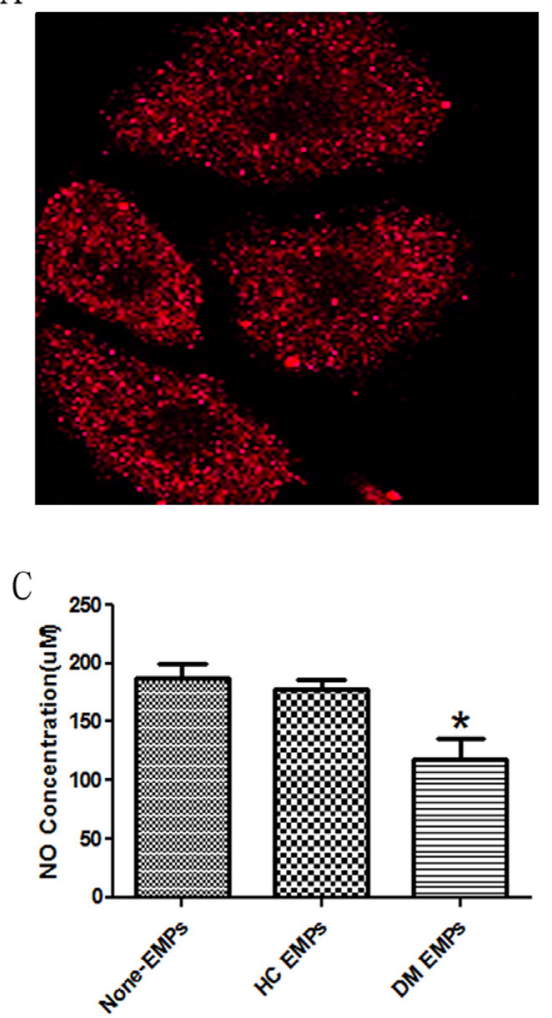

B

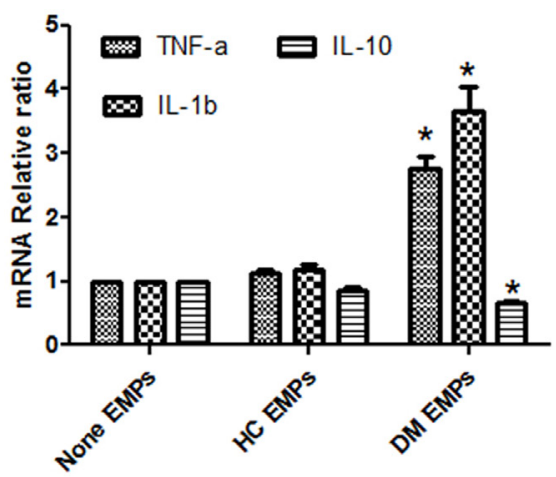

Figure 2: DM patient-derived EMPs induce HUVEC dysfunction. (A) HUVECs effectively engulf EMPs using confocal. Red, PE-labelled EMPs (600x). (B) DM patient-derived EMPs, but not healthy control-derived EMPs, increase the expression of TNF- $a$ and $I L$ $1 b$ mRNA, whereas they decrease the expression of $I L-10$ mRNA. (C) DM patient-derived EMPs, but not healthy control-derived EMPs, decrease NO expression in HUVECs. Healthy control, $\mathrm{n}=6$; and DM, $\mathrm{n}=10$. HC EMPs, healthy control-derived endothelial microparticles; and DM patient-derived EMPs, diabetes mellitus patient-derived endothelial microparticles. ${ }^{*} \mathrm{p}<0.05$. GAPDH mRNA serves as an internal control. 
Sirt6 siRNA. As shown in Figure 4G and Supplementary 1C, Sirt6 mRNA-enriched EMPs partially rescued Sirt6 protein expression in Sirt6 KD HUVECs. Additionally, to exclude whether these effects were caused by free Sirt6 protein, which is centrifuged together with EMPs, or Sirt6 proteins which is contained in EMPs, we lysed the EMPs precipitate directly and then measured using ELISA. Interestingly, the Sirt6 levels were undetectable (Figure 4H).

Undisputedly, the primary nucleotides species found and the most popular research focused in EMPs are microRNAs (miRs) [5], therefore we first using Drosha siRNA in cultured-HUVECs to impede miRs package, then added Sirt6 siRNA or Adv-Sirt6 into these cells to generate EMPs. After careful collection, these miRs-deficient EMPs were added to cultured-HUVECs. Interestingly, Sirt6 $m R N A$, which is incorporated in the EMPs, rescued the ECs dysfunction caused by high glucose, including decreased inflammatory cytokine production and increased NO production, still preserved (Figure 5A, 5B). Additionally, we also packaged Sirt6 $m R N A$ into EMPs using mRNA electroporation method. As shown in Figure 5C-5D, Sirt6 $m R N A$ can effectively transferred into EMPs and then conveyed to target HUVECs. We also observed the inflammatory factors release and NO secretion. As shown in Figure 5E, EMPs packaged Sirt6 $m R N A$ inhibited $I L-1 b$ and $T N F-a$ release but induced $I L-10$ release. Also the NO expression is increase following Sirt6-enriched EMPs treatment (Figure 5F).

\section{eNOS expression differed in HUVECs following exposure to EMPs}

To verify that Sirt6 $m R N A$ did not degrade after transfection, we evaluated Sirt6 protein expression in HUVECs following EMP administration in spite of miRs elimination (using Drosha siRNA) or mRNA electroporation (Figure 6A-6B and Supplementary Figure $1 \mathrm{E}-1 \mathrm{~F})$. Then, we evaluated the activation of eNOS, which is a key mediator in the Sirt6 pathway. Immunoblotting analysis revealed decreased phosphorylation of eNOS when HUVECs were exposed to DM patient-derived EMPs compared with exposure to healthy volunteerderived EMPs, suggesting that eNOS is inactive following stimulation by DM patient-derived EMPs (Figure 6C and Supplementary Figure 1G). The effect of Sirt6 $m R N A$-containing EMPs on eNOS was further confirmed. The relative amount of p-eNOS following Sirt6 $m R N A$-enriched EMP exposure was significantly increased, whereas Sirt6 $m R N A$-deficient EMPs mediated a significant downregulation of eNOS phosphorylation (Figure 6D-6F and Supplementary Figure 1H-1J). These data indicated that eNOS phosphorylation may participate in EMP-mediated EC dysfunction in DM.

\section{Sirt6 $m \boldsymbol{R} N \boldsymbol{A}$-containing EMPs improved ECs function is independent of Sirt1-dependent manner}

Previous documents proven that Sirt1-dependent mechanism was wide agreement in DM-associated
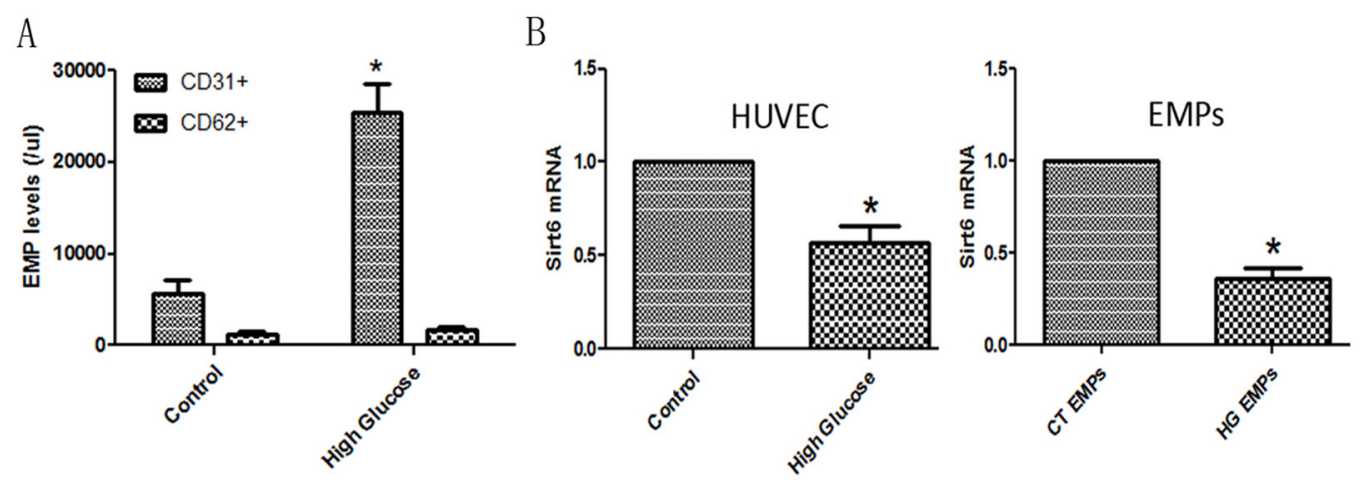

C
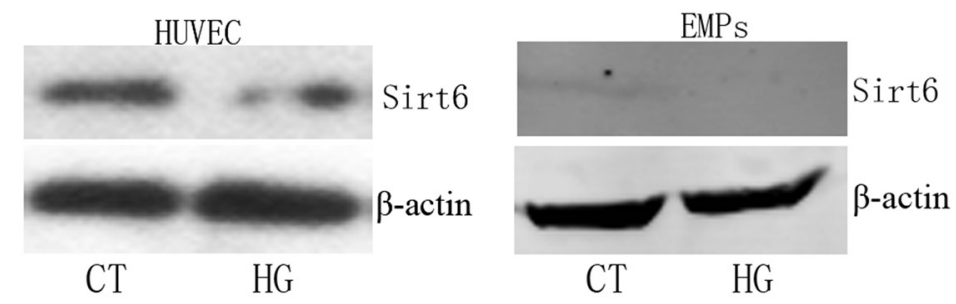

Figure 3: EMP levels and Sirt6 expression in high glucose-cultured HUVECs. (A) High glucose induces the formation of apoptotic (CD31+) EMPs but not activated (CD62+) EMPs. (B) High glucose decreases Sirt6 mRNA expression in HUVECs and EMPs. (C) High glucose decreases Sirt6 protein expression in HUVECs but not EMPs. n=3. EMPs, endothelial microparticles; CT, control; and HG, high glucose. ${ }^{*} \mathrm{p}<0.05$. GAPDH mRNA serves as an internal control. 
A

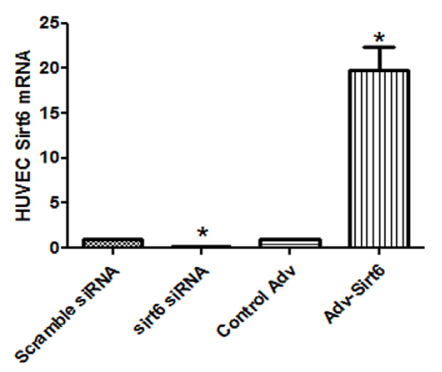

D

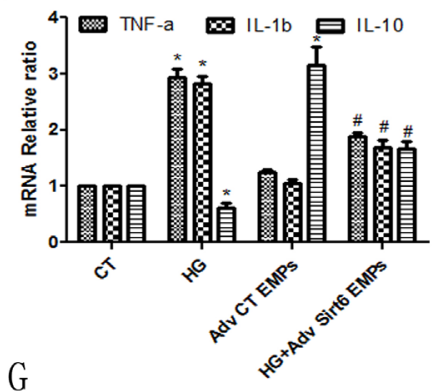

G

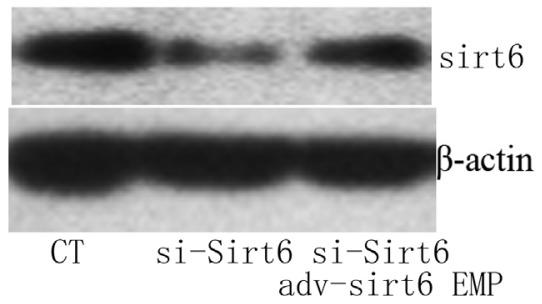

B

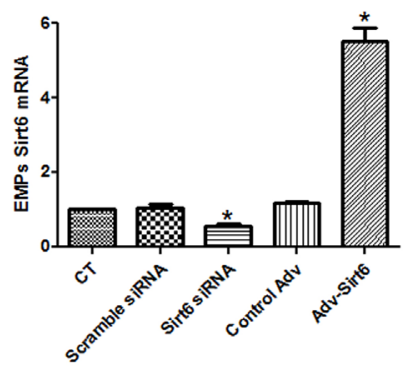

E

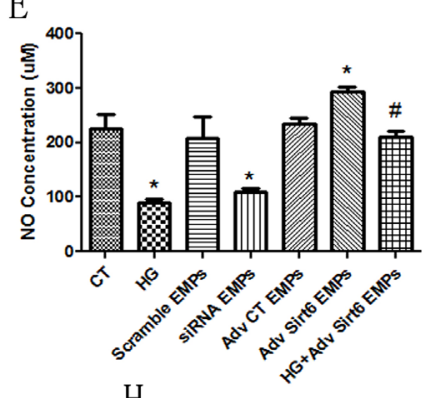

C

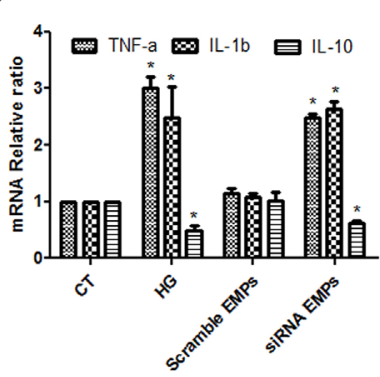

F

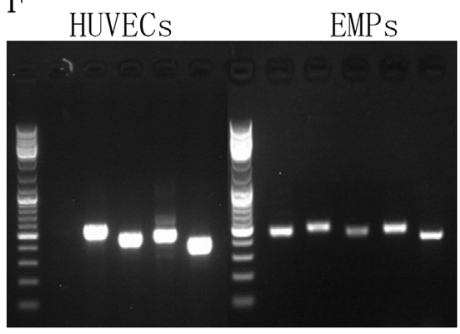

$\mathrm{H}$

I
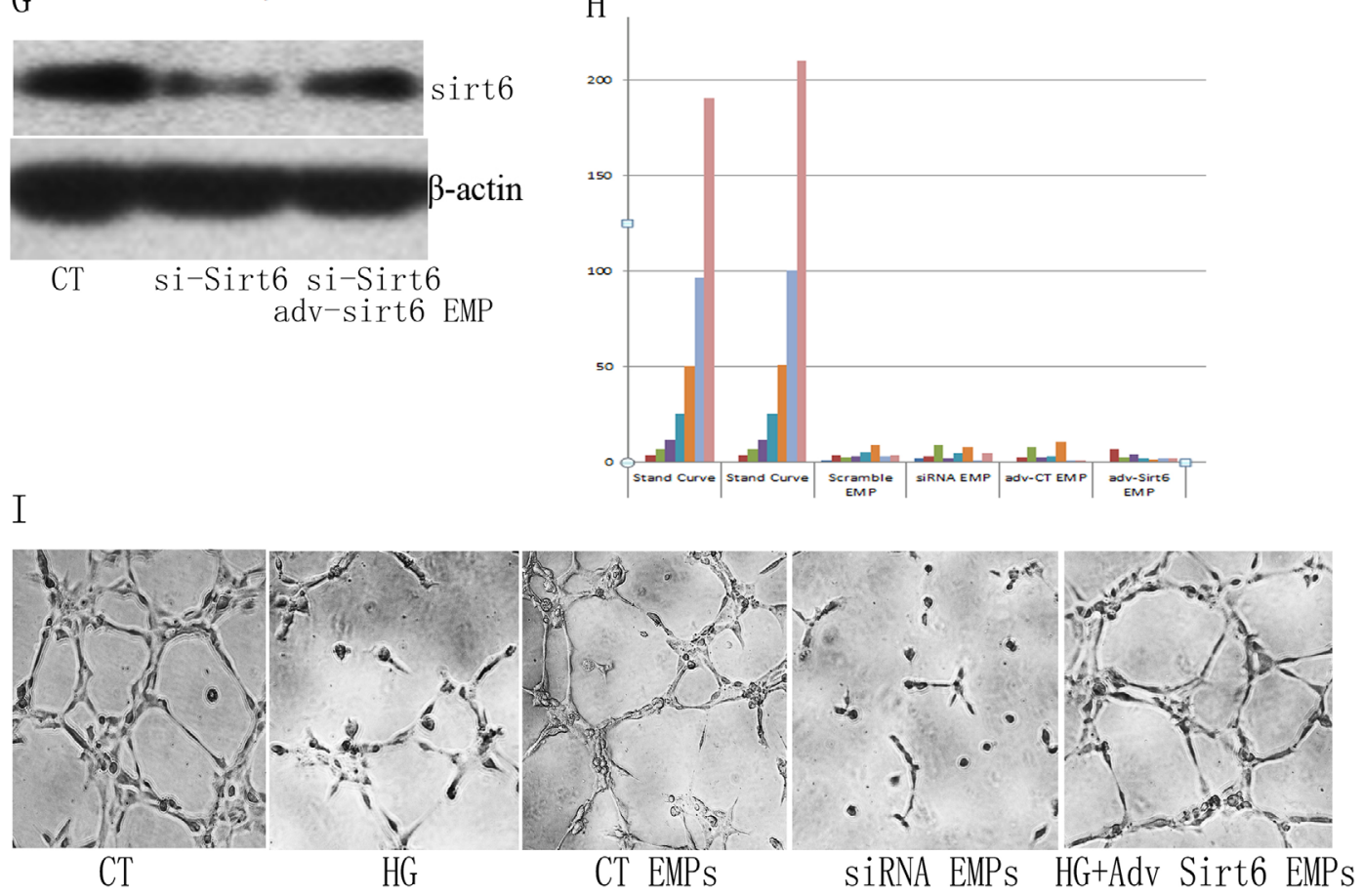

Figure 4: Sirt6 mRNA plays important roles in high glucose-derived EMP-induced HUVEC dysfunction. (A) Sirt6 siRNA or $A d v$-Sirt6 alters Sirt6 mRNA levels in HUVECs. (B) Sirt6 siRNA or Adv-Sirt6 alters Sirt6 mRNA levels in EMPs. (C) Sirt6 siRNA-treated EMPs mimic high glucose-induced $I L-10$ decreases and $T N F$ - $a$ and $I L-1 b$ increases. (D) $A d v$-Sirt6-treated EMPs protect against high glucose-induced $I L-10$ decreases and $T N F$ - $a$ and $I L-1 b$ increases. (E) Sirt 6 siRNA-treated EMPs mimic high glucose-induced NO decreases, whereas $A d v$-Sirt6-treated EMPs protect against high glucose-induced NO decreases. (F) PCR of Sirt6 mRNA incorporated in EMPs and HUVEC. (G) $A d v$-Sirt6 EMPs rescues Sirt6 protein expression in Sirt6 KD HUVECs. si-Sirt6, Sirt6 siRNA. (H) Sirt6 protein in EMPs is undetectable using ELISA. (I) High glucose- and Sirt6 siRNA-treated EMPs impair HUVEC tube formation, whereas $A d v$ Sirt6-treated EMPs induce tube formation in high glucose-cultured HUVECs. n=3. CT, control; HG, high glucose; EMPs, endothelial microparticles; siRNA EMP, Sirt6 siRNA treated-EMP; Adv Sirt6 EMP, adv-Sirt6 treated-EMP. ${ }^{*} \mathrm{p}<0.05$ vs CT; ${ }^{*} \mathrm{p}<0.05$ vs HG. GAPDH mRNA serves as an internal control. "CT EMPs" means that EMPs sourced HUVECs did not undergo siRNA- or adv- contamination. 
endothelial dysfunction [19], therefore we added Sirt6 $m R N A$ - containing EMPs into Sirt1 deficient HUVECs (Figure 7A, 7B). We found that the $I L-1 b$ and $T N F a$ mRNAs were decrease, whereas $I L-10$ mRNA was increase (Figure 7C). Additionally, NO production was induced following Sirt6 mRNA-enriched EMPs treatment (Figure 7D). These data indicated that Sirt6 $m R N A$-containing EMPs improved ECs function is independent of, at least partially, Sirt1-dependent manner.
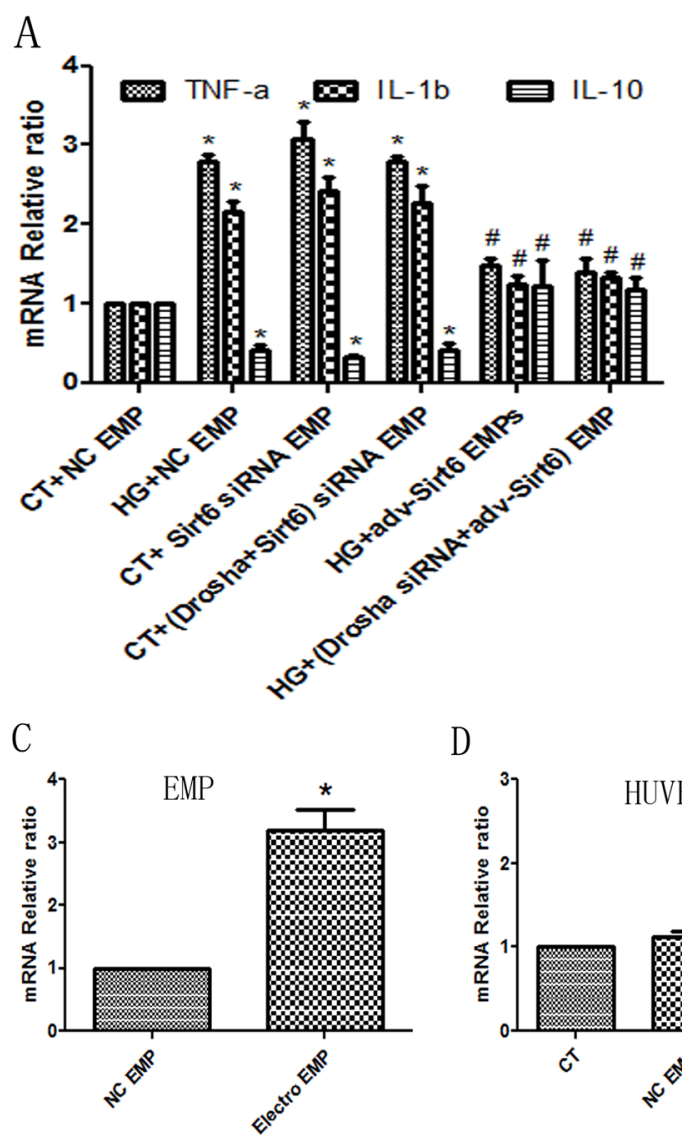

F
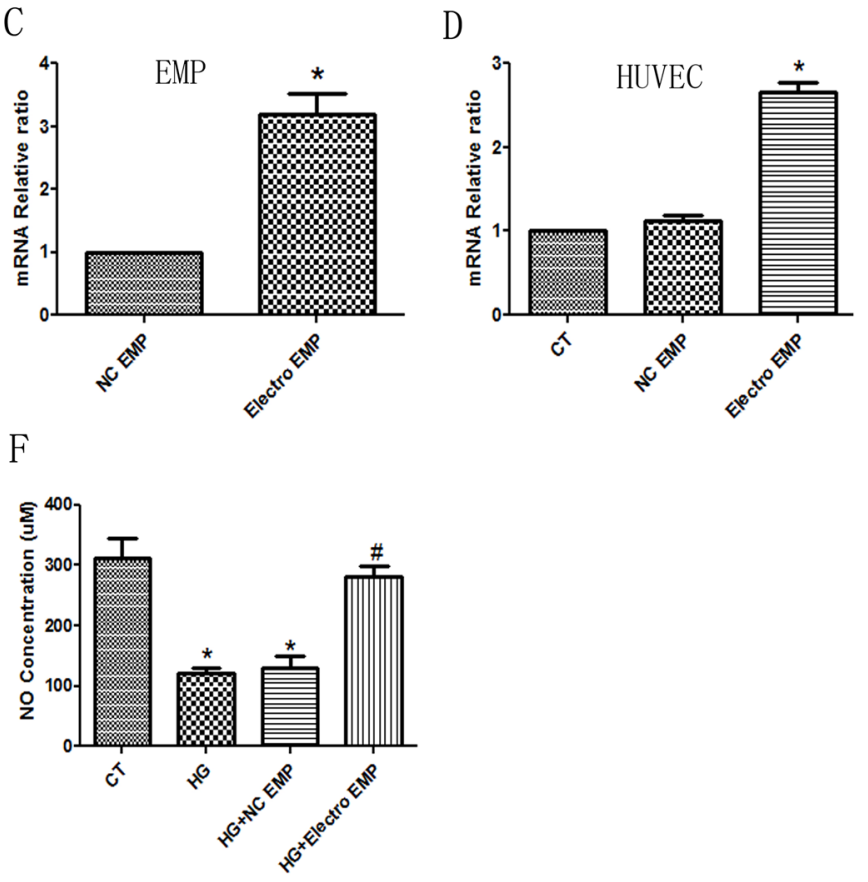

B

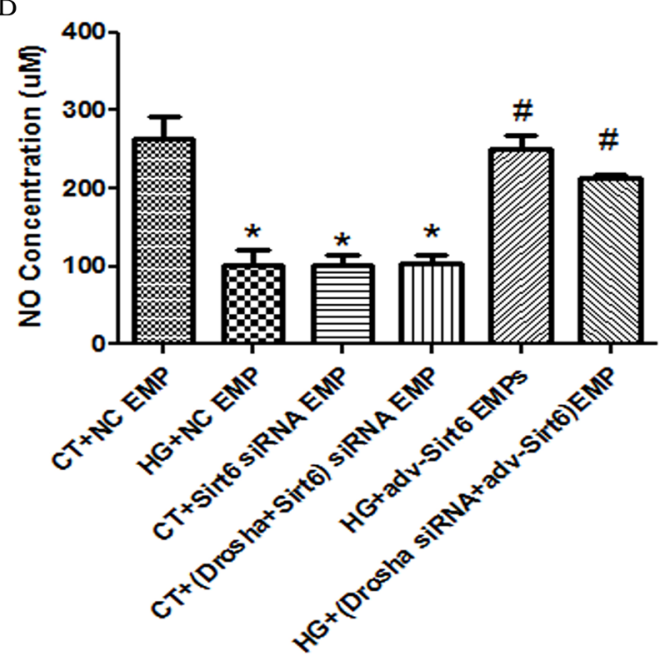

E

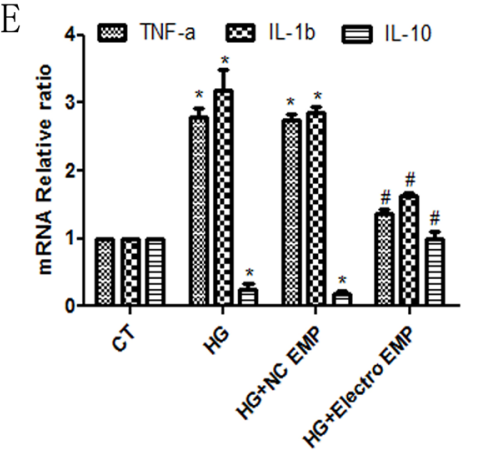

Figure 5: Sirt6 $\boldsymbol{m R \boldsymbol { R } A}$ incorporated in EMPs is a key factor for HUVEC function. Drosha siRNA was used to generate miRsdeficient EMPs. (A) Sirt6 siRNA-treated EMPs mimic high glucose-induced $I L-10$ decreases and $T N F$ - $a$ and $I L-1 b$ increases whether Drosha siRNA presence or absence in EMP formation. Whereas $A d v$-Sirt6-treated EMPs protect against high glucose-induced $I L-10$ decreases and $T N F-a$ and $I L-1 b$ increases whether Drosha siRNA presence or absence in EMP formation. (B) Sirt6 siRNA-treated EMPs mimic high glucose-induced NO decreases, whereas $A d v$-Sirt6-treated EMPs protect against high glucose-induced NO decreases whether Drosha siRNA presence or absence in EMP formation. (C) Electroporation transfers Sirt6 mRNA into EMPs. (D) Eletroporation-EMP effectively transfers Sirt6 $m R N A$ into target HUVECs. (E) Electroporation-EMPs protect against high glucose-induced $I L-10$ decreases and $T N F-a$ and $I L-1 b$ increases. (F) Electroporation-EMPs protect against high glucose-induced NO decreases. n=3. EMPs, endothelial microparticles; CT, control; and HG, high glucose; Electro, electroporation. ${ }^{*} \mathrm{p}<0.05$ vs CT; ${ }^{*} \mathrm{p}<0.05$ vs HG. GAPDH mRNA serves as an internal control. 


\section{DISCUSSION}

The present study demonstrated that DM-derived EMPs activate inflammatory cytokines and decrease the production of NO in cultured HUVECs, thereby facilitating EC damage. Notably, EMPs carrying Sirt6 $m R N A$ are critically involved in the process, and regulation of EMPs containing Sirt6 could effectively affect EMP-mediated inflammatory cytokine activation, NO decrease and impaired tube formation. In addition, the phosphorylation of eNOS was revealed to be associated with the stimulation of ECs by EMPs.

The potential role of endothelial dysfunction in cardiovascular complications has recently been investigated using cultured ECs and experimental models of DM. Indeed, endothelial dysfunction manifests as endothelial erosion, apoptosis, inflammation and premature senescence with pronounced EMP release $[10,18]$. Therefore, the possibility that dysfunctional endothelial cells are a major source of deleterious EMPs in DM patients was evaluated. The present findings further confirmed these previous hypotheses, showing that both EMPs released from activated endothelial cells and apoptotic endothelial cells are upregulated in DM patients, whereas the appearance of a pronounced CD31+/CD42-, apoptotic EMP profile, but not a CD62+/CD42-, activated EMP profile, is observed in high glucose-exposed HUVECs. Notably, the prevalence of CD31+/CD42EMPs is much higher in DM patients than CD62+/CD42EMPs. In addition to representing a surrogate diagnostic and therapeutic marker of endothelial dysfunction, EMPs might also contribute to aggravating the function of circulating ECs, as EMPs from patients with DM exerted prothrombotic, proinflammatory, disseminated
A

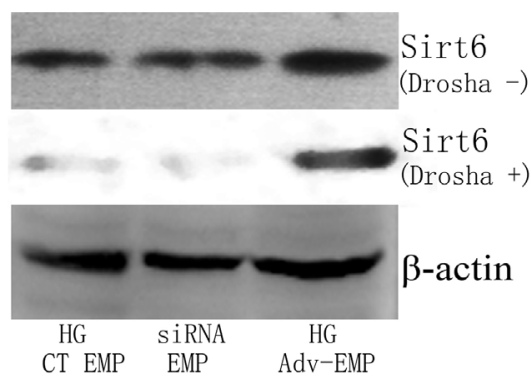

C

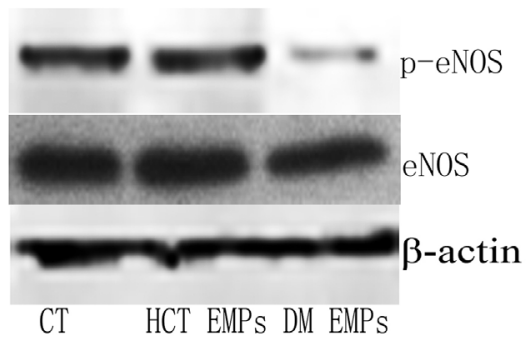

E

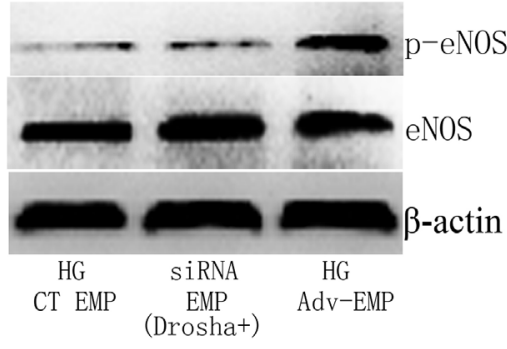

B

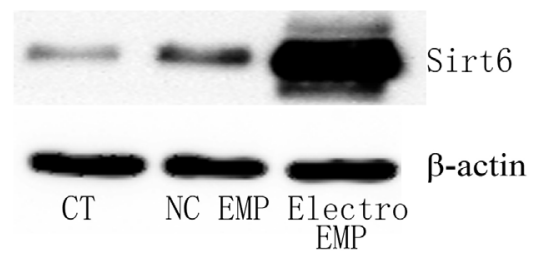

D

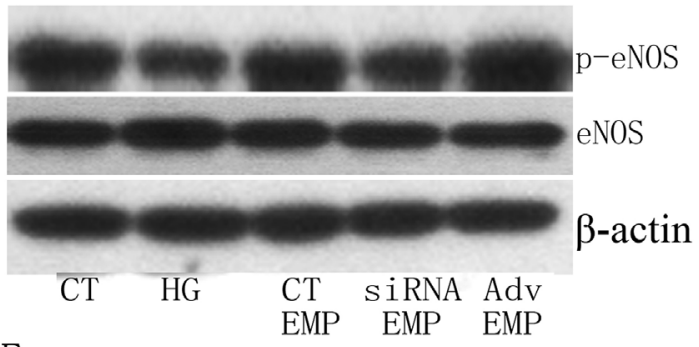

$\mathrm{F}$

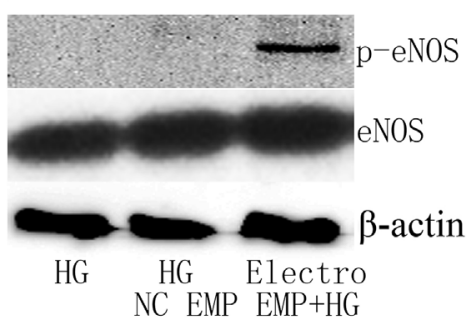

Figure 6: Sirt6 protein and phosphorylation of eNOS is different in HUVECs following exposure to EMPs. (A) Sirt6 $m R N A$-incorporated EMPs, in spite of miRs-enrichment (Drosha-) or miRs-deficient (Drosha +), alter Sirt6 protein expression in HUVECs. (B) Electroporation-EMPs alter Sirt6 protein expression in HUVEC. (C) DM patient-derived EMPs, but not healthy control-derived EMPs, cause a decrease in p-eNOS in HUVECs. (D) High glucose- and Sirt6-siRNA-treated EMPs decease p-eNOS levels, whereas Adv-Sirt6treated EMPs increase p-eNOS levels in HUVECs following high glucose administration. (E) Sirt6 $m R N A$-incorporated EMPs, which are miRs-deficient, induce p-eNOS increase in HUVECs. (F) Sirt6 $m R N A$ electroporation-EMPs increases p-eNOS levels in HUVECs following high glucose administration. $n=3$. CT, control; HCT, healthy control; HG, high glucose; DM, diabetes mellitus; EMPs, endothelial microparticles; Electro, electroporation. 
intravascular coagulopathic, microvascular injury and immunosuppressive effects on circulating ECs [20]. They also blunted endothelium-dependent relaxation in arterial rings [21, 22] and promoted premature endothelial senescence and impaired tube formation in cultured ECs [21]. At present, the uptake of EMPs from DM patients or high-glucose incubated HUVECs increases proinflammatory cytokine release, reduces NO release and impairs tube formation, whereas a similar concentration of EMPs from healthy volunteers are inactive on ECs. Altogether, these findings support the concept that DM patient-derived EMPs carry a pro-damage message to neighboring ECs.

In addition to classical short-distance intercellular communication via signaling molecules and long-distance communication via hormones, extracellular vesicles, such as microparticles and exosomes, have been proposed to be essential for information exchange that affects the physiology of neighboring and remote recipient cells $[23,24]$. Mostly evidence suggests that the information transferred and then elicited pathophysiological effects on the target cells by microparticles and exosomes are mediated by microRNA or protein-dependent mechanisms [25, 26]. Recently, researchers found that mRNA incorporated into MPs also played key roles in tumor biology and predicted prognostic risk stratification [27]. However, there is no doubt that the cargo that is incorporated into the EMPs is dependent on the stimulus received by the parent ECs [28]. miR126, membrane-associated proteins and glycoproteins that are incorporated into the EMPs are accepted as the major mechanism by which information is transferred to neighboring and remote recipient cells $[22,29]$. Sirt6 is a sirtuin family member that participates in the control of a wide range of pathophysiological processes, including glucose homeostasis, cardiovascular diseases, cancer and longevity [30]. The relationship between Sirt6 and diabetes is complex, although Sociali G. reported that pharmacological Sirt6 inhibition improved glucose tolerance as well as reduced triglycerides and cholesterol levels in a T2DM mouse model [31]; most models have confirmed the hypothesis that Sirt6 serves as a surrogate diagnostic and therapeutic marker of DM with or without cardiovascular complications. Sirt6 expression decreases in diabetic atherosclerotic plaque and endothelium [32], whereas overexpression of Sirt6 protects against endothelial senescence [17], inhibits macrophage-derived foam cell formation [33], represses aging [34], delays pancreatic $\beta$-cell dysfunction and apoptosis [35], and enhances insulin sensitivity [36]. Downregulation of Sirt6 accelerates ROS- or high glucose-induced endothelial senescence [17, 37] and leads to obesity and insulin resistance [38]. At present, we also determined that serum Sirt6 levels are much lower in diabetic patients than in healthy controls. However, there is limited information available regarding Sirt6 expression in EMPs from DM patients. Interestingly, the expression of Sirt6 $m R N A$, but not protein, in the EMPs from DM patients and high glucose-incubated HUVECs is consistently decreased; additionally, Sirt6 $m R N A$ and protein deficiency has
A
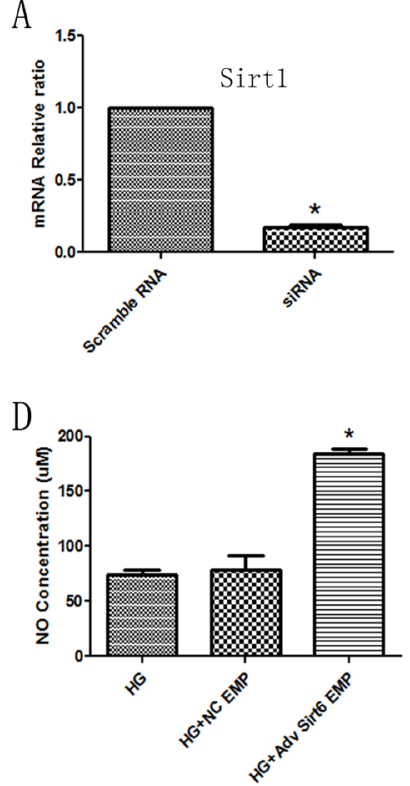

$\mathrm{B}$

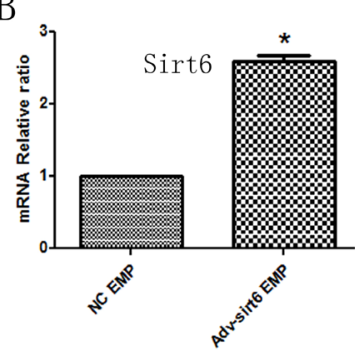

$\mathrm{C}$

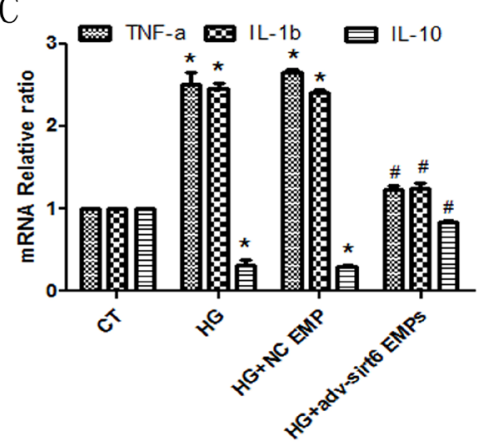

Figure 7: Sirt6 $\boldsymbol{m} \boldsymbol{R} \boldsymbol{N A}$-mediated protective effects are independent of Sirt1. (A) Sirt1 siRNA abrogates Sirt1 mRNA expression in HUVECs. (B) $A d v$-Sirt6-treated EMPs increase Sirt6 $m R N A$ levels in Sirt1 KD HUVECs. (C) Adv-Sirt6-treated EMPs protect against high glucose-induced $I L-10$ decreases and $T N F-a$ and $I L-1 b$ increases in Sirt 1 KD HUVECs. (D) Adv-Sirt6-treated EMPs protect against high glucose-induced NO decreases in Sirt1 KD HUVECs. CT, control; HG, high glucose; EMPs, endothelial microparticles. ${ }^{*}$ p $<0.05$ vs CT; ${ }^{\#} \mathrm{p}<0.05$ vs HG. GAPDH mRNA serves as an internal control. 
Table 1: Oligonucleotide sequences of the primers and siRNAs

\begin{tabular}{|c|c|c|}
\hline Genes & Oligonucleotide sequences & Protocols \\
\hline \multicolumn{3}{|l|}{ Primers } \\
\hline \multirow[t]{2}{*}{$T N F-a$} & Forward: 5'-CCGTCTCCTACCAGACCAAGG-3' & \\
\hline & Reverse: 5'-CTGGAAGACCCCTCCCAGATAG-3' & \\
\hline \multirow[t]{2}{*}{$I L-1 b$} & Forward: 5'-CTGATGGCCCTAAACAGATGAAG-3' & \\
\hline & Reverse: 5'-GGTCGGAGATTCGTAGCAGCTGGAT-3' & \\
\hline \multirow[t]{2}{*}{$I L-10$} & Forward: 5'-CATGCTGCTGGGCCTGAA-3' & \\
\hline & Reverse: 5'-CGTCTCCTTGATCTGCTTGATG-3' & \\
\hline \multirow[t]{2}{*}{ Sirt6 } & Forward: 5'-GCCCCGCTTCCGGCGGAAG-3' & \\
\hline & Reverse: 5'-ATTGTTTTTATTGCATTGAGGAC-3' & \\
\hline \multirow[t]{2}{*}{ Drosha } & Forward: 5'-TCCCTGCTGCCCAAGATTTC-3' & stage 1: $95^{\circ} \mathrm{C}, 30 \mathrm{sec}$ \\
\hline & Reverse: 5'-CAGGGCTTTGCTGCACCTTA-3' & stage $2: 95^{\circ} \mathrm{C}, 5 \mathrm{sec}$ \\
\hline \multirow[t]{2}{*}{ Sirt1 } & Forward: 5'-TGTGGTAGAGCTTGCATTGATCTT-3' & $55^{\circ} \mathrm{C}, 30 \mathrm{sec}$ \\
\hline & Reverse: 5'-GGCCTGTTGCTCTCCTCATT-3' & $72^{\circ} \mathrm{C}, 35 \mathrm{sec}$ \\
\hline \multirow[t]{2}{*}{ GAPDH } & Forward: 5'-AAGGTGAAGGTCGGAGTCA-3' & 40 cycles \\
\hline & Reverse: 5'-GGAAGATGGTGATGGGATTT-3' & \\
\hline \multicolumn{3}{|l|}{ siRNA } \\
\hline \multirow[t]{2}{*}{ Sirt6 } & Sense: 5'-CCCUGGUCUCCAGCUUAAATT-3' & \\
\hline & Anti-Sense: 5'-UUUAAGCUGGAGACCAGGGTT-3' & \\
\hline \multirow[t]{2}{*}{ Sirt1 } & Sense: 5'-GATTATTGCCGGAAACAA-3' & \\
\hline & Anti-Sense: 5'-TCCTTTCAGAACCACCAAA-3' & \\
\hline \multirow[t]{2}{*}{ Drosha } & Sense: 5'-GGATTAGCAACCTATCGGA-3' & \\
\hline & Anti-Sense: 5'-AAGGACCAAGTATTCAGCA-3' & \\
\hline \multirow[t]{2}{*}{ GAPDH: } & Sense: 5'-GUAUGACAACAGCCUCAAGTT-3' & \\
\hline & Anti-Sense: 5'-CUUGAGGCUGUUGUCAUACTT-3' & \\
\hline
\end{tabular}

also been confirmed in HUVECs following high glucose treatment in our study, suggesting that Sirt6 might have significant effects on the pathogenesis of diabetic complications. Additionally, altering Sirt6 $m R N A$ concentrations in EMPs using Adv-Sirt6, Sirt6 siRNA or direct mRNA-electroporation can consistently direct EMP-mediated effects on target HUVECs, including an inflammatory phenotype, NO production and tube formation. These effects also exist using miRs-deficient EMPs. Combining these data, we may deduce that Sirt6 $m R N A$-incorporated EMPs may mediate an important mechanism in the transfer of information to neighboring and remote ECs in diabetes.

It is well known that eNOS-derived NO formation plays a very important role in maintaining endothelial functional integrity. Indeed, both senescent and apoptotic ECs are observed with a pronounced downregulation of eNOS expression and $\mathrm{NO}$ formation
[18, 39]. Recently, researchers have observed that EMPs from CAD or CHF patients inhibit angiogenesis and induce inflammation in ECs via disturbed eNOS phosphorylation and NO formation [40, 41]. The present findings further extend these previous reports by showing that blunted eNOS phosphorylation and NO formation are observed in HUVECs following exposure to EMPs from DM patients or high glucoseincubated ECs, suggesting that the eNOS pathway is very important for the transfer of information via EMPs in DM. Whether the EMP-mediated decrease of eNOS phosphorylation and NO formation is related to Sirt6 is still unclear. Although Liu R reported that the downregulation of Sirt6 decreased eNOS phosphorylation and played key roles in oxidative stress-induced EC senescence [37], the results contrasted with those from Freitas $M$ who showed that Sirt3 and Sirt7, but not Sirt6, had a close relationship 
with eNOS phosphorylation and NO formation in aged patients [32]. At present, our findings further extend these previous reports by showing that blunted eNOS phosphorylation and NO formation can be observed in target ECs following Sirt6-deficient EMP exposure, whereas Sirt6 $m R N A$-overexpressing EMPs cause significant eNOS phosphorylation and NO formation. Thus, it may be possible that Sirt6 deficiency in DM patient-derived EMPs induces endothelial dysfunction via the eNOS pathway.

There is a limitation in this study that should be noted. Although we have shown that Sirt6 $m R N A$ incorporated EMPs are very important for the maintenance of neighboring and remote EC function, the mechanism underlying the effects of Sirt6 on the eNOS pathway has not been completely elucidated. Additionally, direct eNOS regulation in target HUVECs was not shown in this study. Therefore, additional in vivo and ex vivo studies are needed to verify the abovementioned mechanisms.

In conclusion, diabetes leads to increased EMP release and decreased serum Sirt6 levels. Sirt6 $m R N A$ incorporated EMPs are decreased in DM patients and high glucose-incubated HUVECs. EMPs containing Sirt6 $m R N A$ inhibits inflammatory cytokine release and induces tube formation, whereas deficiency of Sirt6 $m R N A$ in EMPs amplifies inflammation and impairs tube formation. Moreover, eNOS phosphorylation and NO formation may be involved in this progress. Many questions must be answered regarding the molecular network underlying these responses. However, the possibility that the Sirt6 $m R N A$-incorporated EMPs have an essential effect on neighboring and remote ECs in DM is very exciting.

\section{MATERIALS AND METHODS}

\section{Reagents}

Glucose and TRIzol were purchased from Sigma (St Louis, Missouri, USA). Monoclonal antibodies against CD62-PE, CD31-PE, CD42-FITC and IgGPE were obtained from BD (Shanghai, China), and the monoclonal rabbit antibodies against Sirt6, p-eNOS, eNOS and $\beta$-actin were obtained from Cell Signaling (Denver, Colorado, USA). The fetal bovine serum (FBS), DMEM culture medium with or without glucose, Lipofectamine ${ }^{\circledR} \quad 2000$ Transfection Reagent and ECMatirm $^{\mathrm{TM}}$ solution were purchased from Invitrogen (Carlsbad, CA, USA). The cDNA Synthesis Kit and Premix Ex Taq SYBR Green PCR Kit were purchased from Takara (Shiga, Japan). The $a d v$-Sirt6, Sirt6 siRNA, $C M V$-Sirt6 shRNA, Sirt1 siRNA and Drosha siRNA were obtained from HanBio. (Shanghai, China). Other unmentioned reagents were purchased from Shenggong Bio. (Shanghai, China).

\section{Patients and blood collection}

Informed consent was obtained from all participants in accordance with the guidelines of the Human Subjects Committee of the Medical Ethics Commission of the Tongji Hospital Affiliated with Tongji University (Shanghai, China). Between August 2014 to March 2015, 10 diabetes patients without hypertension, smoking, chronic kidney disease, peripheral atherosclerosis, inflammation, malignant tumors, severe hepatic dysfunction or coronary atherosclerosis who were diagnosed by coronary angiogram at our inpatient department were screened for inclusion in the study. Six age-matched healthy volunteers were also included in our present study. All DM patients were treated with either oral antidiabetic drugs or subcutaneous insulin administration.

Venous blood was obtained from the cubital vein under sterile conditions and then buffered with sodium citrate (for EMP analysis) or with anticoagulant sodium (for Sirt6 quantification). Blood was centrifuged at $1,500 \mathrm{~g}$ for $10 \mathrm{~min}$, and then, the supernatant was centrifuged at 13,000 g for $20 \mathrm{~min}$ to obtain MPs, which were subsequently resuspended in PBS. The blood was coagulated for $45 \mathrm{~min}$, and then, the serum was collected to measure Sirt6 levels.

\section{Cell culture}

The human umbilical vein endothelial cell (HUVEC) line and endothelial cell media (ECM) were obtained from ScienCell (Carlsbad, California, USA). The HUVECs were grown in ECM (glucose: $5.5 \mathrm{mM}$ ), starved by culturing them without FBS for $12 \mathrm{~h}$ and then exposed to glucose at concentrations of $0 \mathrm{mM}$ for $24 \mathrm{~h}$ or $33 \mathrm{mM}$ for $72 \mathrm{~h}$ to generate EMPs. Additionally, Adv-Sirt6 or Sirt6 siRNA was used to alter EMP components.

\section{Adv-Sirt6 transfection}

$A d v$-control or $A d v$-Sirt6 was directly added to cultured cells and incubated for $24 \mathrm{~h}$.

\section{siRNA transfection}

Cells were transfected with either scramble siRNA, Sirt6 siRNA, Sirt1 siRNA or Drosha siRNA using Lipofectamine ${ }^{\circledR} 2000$ Transfection Reagent following the manufacturer's procedure. The siRNA sequence is shown on Table 1.

\section{EMP collection from HUVECs}

After incubation with $A d v$-Sirt6, Sirt6 siRNA or Drosha siRNA for $24 \mathrm{~h}$, the supernatant was removed, and the cells were carefully washed with PBS 3 times 
for $5 \mathrm{~min}$. Then, the cells were exposed to glucose at concentrations of $0 \mathrm{mM}$ for $24 \mathrm{~h}$ or $33 \mathrm{mM}$ for $72 \mathrm{~h}$. The medium was collected and centrifuged at $1000 \mathrm{rpm}$ for 5 min, and then, the supernatant was centrifuged at 13000 $\mathrm{g}$ for $20 \mathrm{~min}$ to obtain MP, which were resuspended in PBS.

\section{Quantitative determination of the plasma EMP levels or cultured HUVEC EMP levels}

As previous description, CD31 and CD62 were biomarkers of endothelial cells, whereas CD42 was biomarker of platelets, therefore, CD62+/CD42- or CD31+/CD42- were considered to be EMPs in plasma. Usually, documents reported that CD62+ EMPs released from activated endothelial cells, whereas CD31+ released from apoptotic endothelial cells [2]. CD31+/CD42- or CD62+/CD42- MPs levels were measured freshly with flow cytometry by using CD42-FITC and CD31-PE/ CD62-PE.

\section{Real-time reverse transcription PCR (RT-PCR)}

Total RNA from HUVECs or MPs (obtained using the TRIzol procedure) was converted to cDNA using Takara reverse transcriptase. RT-PCR was performed using the samples and SYBR Premix regent on an ABI 7500. The primers (Shenggong Bio., China) are displayed on Table 1

\section{RNA-seq}

Total RNA from HUVECs or MPs was tested for quality and treated with DNase and RiboZero Kits (Illumina, Singapore) to remove DNA and rRNA. RNAseq was performed by Shenggong Bio., China.

\section{mRNA electroporation}

After purified of mRNA using Oligotex mRNA Mini Kit (Qiagen, Germany), the Sirt6 $m R N A$ was executed of electroporation into EMPs was performed using GenePulser XcellTM electroporation system (BioRad, USA) as previously described [42]. Briefly, $1 \times 10^{5}$ EMPs and $4 \mathrm{ug}$ mRNA were mixed in $200 \mathrm{ul}$ of electroporation buffer and electroporated at $350 \mathrm{~V}$ and $150 \mathrm{uF}$ in a 10 msec. After incubated at $37^{\circ} \mathrm{C}$ for $30 \mathrm{~min}$, the EMPs were transferred to cultured cells.

\section{Western blotting}

Equal amounts of total protein from HUVEC or MPs (obtained using cell total protein lysis buffer, Beyotime, Haimen, China) were subjected to $8-10 \%$ SDS-PAGE, transferred to PVDF membranes and then blocked using $5 \%$ non-fat milk. After incubation with primary antibodies at $4{ }^{\circ} \mathrm{C}$ overnight, HRP-conjugated secondary antibodies were applied at room temperature for $1 \mathrm{~h}$. Immunoblots were visualized using enhanced chemiluminescence reagents (Bio-Max, Israel).

\section{Enzyme-linked immunosorbent assay of Sirt6 production in blood and EMPs}

Sirt6 production was detected in blood or EMPs using human ELISA kits according to the manufacturer's instructions.

\section{HUVEC tube formation assay in ECMatirm ${ }^{\mathrm{TM}}$ solution}

To assess the vasculogenic effects of EMPs on endothelial cells ex vivo, an ECMatirm $^{\mathrm{TM}}$ solution tube formation assay was performed according to the manufacturer's instructions.

\section{NO assay}

NO levels in culture supernatants were measured using the nitrite method (Jiancheng, Nanjing, China). Briefly, the reaction substrate was mixed with supernatants, vortexed, incubated and then centrifuged at $4,500 \mathrm{rpm}$ for $15 \mathrm{~min}$. The supernatant was then incubated with the chromogenic reagent for $15 \mathrm{~min}$. Finally, the output was detected at an optical absorbance of $540 \mathrm{~nm}$.

\section{Statistical analyses}

Significant differences were determined by oneway ANOVA followed by Tukey's HSD test using SPSS14.0 software, and all data are presented as the mean \pm SEM. A value of $p<0.05$ was considered statistically significant.

\section{Abbreviations}

DM: diabetes mellitus; EMPs: endothelial microparticles; ECs: endothelial cells; Sirt6: sirtuin 6; Sirt1: sirtuin 1; MPs: microparticles; HUVEC: the human umbilical vein endothelial cells; CAD: coronary artery disease; CHF: chronic heart failure.

\section{Author contributions}

CF, LXB, TJ, KYS conceived and designed the study. TJ, KYS, WXJ, HHJ performed the experiments. CF, TJ and KYS wrote the paper. LXB, LY and YYA reviewed and edited the manuscript. All authors have reviewed and approved the manuscript.

\section{ACKNOWLEDGMENTS}

We thank Prof. Fei Zheng for helping us carry out some of the western-blotting tests. 


\section{CONFLICTS OF INTEREST}

None declared.

\section{FUNDINGS}

This work was supported by the China National Natural Science Foundation, Nos: 81370390, 81500381 and 81670403 .

\section{REFERENCES}

1. León LE, Rani S, Fernandez M, Larico M, Calligaris SD. Subclinical detection of diabetic cardiomyopathy with microRNAs: challenges and perspectives. J Diabetes Res. 2016; 2016:6143129.

2. Deng F, Wang S, Zhang L. Endothelial microparticles act as novel diagnostic and therapeutic biomarkers of diabetes and its complications: a literature review. Biomed Res Int. 2016; 2016:9802026.

3. Natali A, Ferrannini E. Endothelial dysfunction in type 2 diabetes. Diabetologia. 2012; 55:1559-1563.

4. Wells BJ, Roth R, Nowacki AS, Arrigain S, Yu C, Rosenkrans WA Jr, Kattan MW. Prediction of morbidity and mortality in patients with type 2 diabetes. Peer J. 2013; $1: \mathrm{e} 87$.

5. Liebner S, Cavallaro U, Dejana E. The multiple languages of endothelial cell-to-cell communication. Aeterioscler Thromb Vasc Bio. 2006; 26:1431-1438.

6. Yuana Y, Sturk A, Nieuwland R. Extracellular vesicles in physiological and pathological conditions. Blood Rev. 2013; 27:31-39.

7. Dignat-George F, Boulanger CM. The many faces of endothelial microparticles. Arterioscler Thromb Vasc Biol. 2011; 31:27-33.

8. Jansen F, Wang H, Przybilla D, Franklin BS, Dolf A, Pferfer P, Schmitz T, Flender A, Endl E, Nickenig G, Werner N. Vascular endothelial microparticles-incorporated microRNAs are altered in patients with diabetes mellitus. Cardiovasc Diabetol. 2016; 15:49.

9. Jansen F, Yang X, Hoelscher M, Cattelan A, Schmitz T, Proebsting S, Wenzel D, Vosen S, Franklin BS, Fleischmann BK, Nickenig G, Werner N. Endothelial microparticlemediated transfer of MicroRNA-126 promotes vascular endothelial cell repair via SPRED1 and is abrogated in glucose-damaged endothelial microparticles. Circulation. 2013; 128:2026-2038.

10. Bernard S, Loffroy R, Sérusclat A, Boussel L, Bonnefoy E, Thévenon C, Rabilloud M, Revel D, Moulin P, Douek P. Increased levels of endothelial microparticles CD144 (VE-Cadherin) positives in type 2 diabetic patients with coronary noncalcified plaqued evaluated by multidetector computed tomography (MDCT). Atherosclerosis. 2009; 203:429-435.
11. Chen J, Chen S, Chen Y, Zhang C, Wang J, Zhang W, Liu G, Zhao B, Chen Y. Circulating endothelial progenitor cells and cellular membrane microparticles in $\mathrm{db} / \mathrm{db}$ diabetic mouse: possible implications in cerebral ischemic damage. Am J Physiol Endocrinol Metab.2011; 301:e62-71.

12. Tsimerman G, Roguin A, Bachar A, Melamed E, Brenner B, Aharon A. Involvement of microparticles in diabetic vascular complications. Thromb Haemost. 2011; 106:310-321.

13. Chironi GN, Boulanger CM, Simon A, Dignat-George F, Freyssinet JM, Tedgui A. Endothelial microparticles in diseases. Cell Tissue Res. 2009; 335:143-151.

14. Tian K, Liu Z, Wang J, Xu S, You T, Liu P. Sirtuin-6 inhibits cardiac fibroblasts differentiation into myofibroblasts via inactivation of nuclear factor $\mathrm{kB}$ signaling. Transl Res. 2015; 165:374-386.

15. Conti V, Forte M, Corbi G, Russomanno G, Formisano L, Landolfi A, Izzo V, Filippelli A, Vecchione C, Carrizzo A. Sirtuins: possible clinical implications in cardio and cerebrovascular diseases. Curr Drug Targets. 2017; 18:473-484.

16. Thandavarayan RA, Garikipati VN, Joladarashi D, Suresh Babu S, Jeyabal P, Verma SK, Mackie AR, Khan M, Arumugam S, Watanabe K, Kishore R, Krishnamurthy P. Sirtuin-6 deficiency exacerbates diabetes-induced impairment of wound healing. Exp Dermatol. 2015; 24:773-778.

17. D’Onofrio N, Servillo L, Giovane A, Casale R, Vitiello M, Marfella R, Paolisso G, Balestrieri ML. Ergothioneine oxidation in the protection against high-glucose induced endothelial senescence: involvement of SIRT1 and SIRT6. Free Radic Biol Med. 2016; 96:211-222.

18. Chen F, Chen B, Xiao FQ, Wu YT, Wang RH, Sun ZW, Fu GS, Mou Y, Tao W, Hu XS, Hu SJ. Autophagy protects against senescence and apoptosis via the RAS-mitochondria in high-glucose-induced endothelial cells. Cell Physiol Biochem. 2014; 33:1058-1074.

19. Kumar S, Kim YR, Vikram A, Naqvi A, Li Q, Kassan M, Bachschmid MM, Jacobs JS, Kumar A, Irani K. Siruin-1 regulated lysine acetylation of p66Shc governs diabetes-induced vascular oxidative stress and endothelial dysfunction. PNAS. 2017; 114:1714-1719.

20. Souza AC, Yuen PS, Star RA. Microparticles: markers and mediators of sepsis-induced microvascular dysfunction, immunosuppression, and AKI. Kidney Int. 2015; 87:1100-1108.

21. Abbas M, Jesel L, Auger C, Amoura L, Messas N, Manin G, Rumig C, León-González AJ, Ribeiro TP, Silva GC, Abou-Merhi R, Hamage E, Hecker M, et al. Endothelial microparticles from acute coronary syndrome patients induce premature coronary artery endothelial cell aging and thrombogenicity: role of the Ang II/AT1 receptor/NADPH oxidase-mediated activation of MAPKs and PI3-kinase pathways. Circulation. 2017; 135:280-296. 
22. Jansen F, Stumpf T, Proebsting S, Franklin BS, Wenzel D, Pferfer P, Flender A, Schmitz T, Yang X, Fleischmann BK, Nickenig G, Werner N. Intercellular transfer of miR-126-3p by endothelial microparticles reduces vascular smooth muscle cell proliferation and limits neointima formation by inhibiting LRP6. J Mol Cell Cardiol. 2107; 104:43-52.

23. Yellon DM, Davidson SM. Exosomes: nanoparticles involved in cardioprotection? Circ Res. 2014; 114:325-332.

24. Hulsmans M, Holvoet P. MicroRNA-containing microvesicles regulating inflammation in association with atherosclerotic disease. Cardiovasc Res. 2013; 100:7-18.

25. Nie DM, Wu QL, Zheng P, Chen P, Zhang R, Li BB, Fang J, Xia LH, Hong M. Endothelial microparticles carrying hedgehog-interacting protein induce continuous endothelial damage in the pathogenesis of acute graft-versus-host disease. Am J Physiol Cell Physiol. 2016; 310:C821-835.

26. Martinez MC, Larbret F, Zobairi F, Coulombe J, Debili N, Vainchenker W, Ruat M, Freyssinet JM. Transfer of differentiation signal by membrane microvesicles harboring hedgehog morphogens. Blood. 2006; 108:3012-3020.

27. Xu JF, Wang YP, Zhang SJ, Chen Y, Gu HF, Dou XF, Xia B, Bi Q, Fan SW. Exosomes containing differential expression of microRNA and mRNA in osteosarcoma that can predict response to chemotherapy. Oncotarget. 2017; 8:7596875978. https://doi.org/10.18632/oncotarget.18373.

28. Peterson DB, Sander T, Kaul S, Wakim BT, Halligan B, Twigger S, Pritchard KA Jr, Oldham KT, Ou JS. Comparative proteomic analysis of PAI-1 and TNF-alphaderived endothelial microparticles. Proteomics. 2008; 8:2430-2446.

29. Burnier L, Fontana P, Kwak BR, Angelillo-Scherrer A. Cell-derived microparticles in haemostasis and vascular medicine. Thromb Haemost. 2009; 101:439-451.

30. Vitiello M, Zullo A, Servillo L, Mancini FP, Borriello A, Giovane A, Della Ragione F, D’Onofrio N, Balestrieri ML. Multiple pathways of SIRT6 at the crossroads in the control of longevity, cancer, and cardiovascular diseases. Ageing Res Rev. 2017; 35:301-311.

31. Sociali G, Magnone M, Ravera S, Damonte P, Vigliarolo T, Von Holtey M, Vellone VG, Millo E, Caffa I, Cea M, Parenti MD, Del Rio A, Murone M, et al. Pharmacological Sirt6 inhibition improves glucose tolerance in a type 2 diabetes mouse model. FASEB J. 2017; Epub.

32. Freitas M, Rodrigues AR, Tomada N, Fonseca J, Magalhães A, Gouveia AM, Neves D. Effects of aging and cardiovascular disease risk factors on the expression of sirtuins in the human corpus cavernosum. J Sex Med. 2015; 12:2141-2152. https://doi.org/10.18632/aging.100975.

33. Breitenstein A, Stein S, Holy EW, Camici GG, Lohmann C, Akhmedov A, Spescha R, Elliott PJ, Westphal CH, Matter CM, Luscher TF, Tanner FC. Sirt1 inhibition promotes in vivo arterial thrombosis and tissue factor expression in stimulated cells. Cardiovasc Res. 2011; 89:464-472.

34. Xu S, Yin M, Koroleva M, Mastrangelo MA, Zhang W, Bai P, Little PJ, Jin ZG. SIRT6 protects against endothelial dysfunction and atherosclerosis in mice. Aging (Albany NY). 2016; 8:1064-1082. https://doi.org/10.18632/aging.100975.

35. Xiong X, Sun X, Wang Q, Qian X, Zhang Y, Pan X, Dong XC. SIRT6 protects against palmitate-induced pancreatic $\beta$-cell dysfunction and apoptosis. J Endocrinol. 2016; 231:159-165.

36. Anderson JG, Ramadori G, Ioris RM, Galiè M, Berglund ED, Coate KC, Fujikawa T, Pucciarelli S, Moreschini B, Amici A, Andreani C, Coppari R. Enhanced insulin sensitivity in skeletal muscle and liver by physiological overexpression of SIRT6. Mol Metab. 2015; 4:846-856.

37. Liu R, Liu H, Ha Y, Tilton RG, Zhang W. Oxidative stress induces endothelial cell senescence via downregulation of Sirt6. Biomed Res Int. 2014; 2014:902842.

38. Kuang J, Zhang Y, Liu Q, Shen J, Pu S, Cheng S, Chen L, Li H, Wu T, Li R, Li Y, Zou M, Zhang Z, et al. Fat-specific Sirt6 ablation sensitizes mice to high-fat diet-induced obesity and insulin resistance by inhibiting lipolysis. Diabetes. 2017; 66:1159-1171.

39. Donato AJ, Morgan RG, Walker AE, Lesniewski LA. Cellular and molecular biology of aging endothelial cells. J Mol Cell Cardiol. 2015; 89:122-135.

40. Ou ZJ, Chang FJ, Luo D, Liao XL, Wang ZP, Zhang X, $\mathrm{Xu}$ YQ, Ou JS. Endothelium-derived microparticles inhibit angiogenesis in the heart and enhance the inhibitory effects of hypercholesterolemia on angiogenesis. Am J Physiol Endocrinol Metab. 2011; 300:E661-668.

41. Lin ZB, Ci HB, Li Y, Cheng TP, Liu DH, Wang YS, Xu J, Yuan HX, Li HM, Chen J, Zhou L, Wang ZP, Zhang X, et al. Endothelial microparticles are increased in congenital heart diseases and contribute to endothelial dysfunction. $\mathrm{J}$ Transl Med. 2017; 15:4.

42. Qu L, Ding J, Chen C, Wu ZJ, Liu B, Gao Y, Chen W, Liu F, Sun W, Li XF, Wang X, Wang Y, Xu ZY, et al. Exosometransmitted lncASAR promotes Sunitinib resistance in renal cancer by acting as a competing endogenous RNA. Cancer Cell. 2016; 29:653-668. 